\title{
Conprehensive Analysis of circRNA-microRNA- mRNA Network Revealed Novel Regulatory Mechanism in Trichosposon Asahii Infection
}

\section{Ming-Wang Zhang}

7th medical center of PLA general hospital https://orcid.org/0000-0002-6517-383X

\section{Zhi-Hong Zhu}

Hainan hospital of Chinese PLA General Hospital

\section{Zhi-Kuan Xia}

the 7th medical center of PLA general hospital

\section{Xin Yang}

the 7th medical center of PLA General Hospital

Wan-Ting Luo

the 7th medical center of PLA general hospital

Jun-Hong Ao

the 7th medical center of PLA general hospital

Rong-Ya Yang ( $\nabla$ yangrya@sina.com )

\section{Research}

Keywords: Trichosporon asahii, circular RNA, competing endogenous RNA, RNA sequencing

Posted Date: June 11th, 2020

DOI: https://doi.org/10.21203/rs.3.rs-33436/v1

License: (c) (1) This work is licensed under a Creative Commons Attribution 4.0 International License.

Read Full License

Version of Record: A version of this preprint was published on March 9th, 2021. See the published version at https://doi.org/10.1186/s40779-021-00311-w. 


\section{Abstract}

Background: Trichosporon asahii (T. asahii) invasive infection frequently occurs in immunodeficient hosts with high mortality, but the pathogenesis of $T$. asahii infection remains elusive. Circular RNAs (circRNAs) are a type of endogenous noncoding RNAs that participant various disease processes. However, the mechanism of circRNAs in $T$. asahii infection are still completely unknown.

Methods: RNA sequencing (RNA-seq) was performed to analyse the expression profiles of circRNA, microRNA (miRNA), and mRNA in THP-1 cells infected with T. asahii or uninfected samples. Part of the RNA-seq results were verified by RT-qPCR. Gene Ontology (GO) enrichment and Kyoto Encyclopedia of Genes and Genomes (KEGG) analysis were used to analyse the differentially expressed (DE) mRNA. The circRNA-miRNA-mRNA network was constructed and verified with dual-luciferase reporter assay and overexpression experiment.

Results: A total of 46 circRNAs , 412 mRNAs and 47 miRNAs were DE after T. asahii infection at $12 \mathrm{~h}$. GO and KEGG analysis showed that the DE mRNAs were primary linked to the leukocyte migration involved in inflammatory response, Toll-like receptor signaling pathway, and TNF signaling pathway. A competing endogenous RNA (ceRNA) network was constructed with five DE circRNAs, five DE miRNAs and 42 DE mRNAs. Among them, we verified that hsa_circ_0065336 indirectly regulate PTPN11 expression by sponging miR-505-3p.

Conclusions: These data revealed a comprehensive circRNA-associated ceRNA network during T. asahii infection, thus providing new insights to clarify the pathogenesis between T. asahii-host interation.

\section{Background}

Trichosporon asahii (T. asahii) is the most common pathogenic species in the genus trichosporon that widely distributed in tropical and temperate regions, usually through built-in catheters, intestinal mucosa translocation of microorganisms or respiratory inhalation to invade the human body[1]. T. asahii are usually associated with superficial mycosis in immunocompetent hosts but could cause invasive infections in immunosuppressed patients. With the abuse of immunosuppressants and broad-spectrum antibiotics and the extensive development of traumatic surgery, the incidence of invasive trichosporonosis has increased over the past years[2]. Despite that various antifungal drugs have been used in the treatment of invasive trichosporonosis, such as amphotericin B, flucytosine, caspofungin and fluconazole, the mortality of invasive trichosporonosis is still high and requires further analysis[3].

Circular RNAs (circRNAs) were a type of endogenous noncoding RNAs that are widely distributed in eukaryotic cells and have the function of regulating gene expression. They are constituted by a covalently closed cyclic structure with high abundance, relative conserved, tissue- specific and cell- specific expression patterns [4]. Although circRNAs have been discovered for more than 40 years, they were mainly regarded as 'junk' generated by mis-splicing of transcripts $[5,6]$. In recent years, with the wide chnology and the rapid development of circRNA- specific 
computational tools, various circRNAs have been reported to be involved in the pathogenesis of cancer, neurological disorders, cardiovascular diseases, diabetes mellitus and autoimmune diseases [7-11]. However, circRNAs associated with fungal infections have not been reported up to date.

MicroRNAs (miRNAs) are small noncoding RNAs that can negatively control their target gene expression post-transcriptionally $[12,13]$. Recent studies have characterized the miRNAs expression profiles and identified several critical miRNAs that participate immune and inflammatory responses following fungal exposure [14]. However, the upstream regulation mechanism of miRNAs are poorly understood. It has been reported that circRNAs can regulate gene expression by binding miRNA response elements (MREs) called competing endogenous RNAs (ceRNAs) mechanism [15]. But whether circRNAs could serve as ceRNAs and involve in the interactions between $T$. asahii and its host remain unknown.

In this study, we systematically investigated the alteration of circRNAs, miRNAs, and mRNAs expression profiles in macrophages during $T$. asahii infection by using RNA-sEq. A large number of dysregulated circRNAs, miRNAs and mRNA were identified after T. asahii infection. CeRNAs network revealed the putative regulatory roles of circRNAs in cellular response to $T$. asahii infection. Furthermore, we identified that hsa_circ_0065336 could indirectly regulate PTPN11 expression by sponging miR-505-3p. In summary, this is the first report of the expression profile and function analyses of circRNAs after fungal exposure in mammalian cell.

\section{Methods}

\section{Cell culture and T. asahii infection}

Human monocyte line THP-1 was cultured with maintained in RPMI 1640 (Gibco, USA) supplemented with $10 \%$ fetal bovine serum (Gibco, USA), $1 \%$ penicillin/streptomycin (Gibco, USA) and $0.05 \mathrm{mmol} / \mathrm{L} \beta$ mercaptoethanol (Amresco, USA) at $37{ }^{\circ} \mathrm{C}$ and $5 \% \mathrm{CO}_{2}$ atmosphere. 293T cells were cultured with $10 \%$ FBS (Gibco, USA) at $37^{\circ} \mathrm{C}$ and $5 \% \mathrm{CO}_{2} .100 \mathrm{ng} / \mathrm{mL}$ Phorbol 12-myristate 13-acetate (PMA) (Sigma, USA) was utilized to induce THP-1 to differentiate into macrophages for $48 \mathrm{~h}$, which was used throughout this study. Heat inactivation T. asahii standard strain CBS2479 was obtained by incubating at $65^{\circ} \mathrm{C}$ for $3 \mathrm{~h}$ in a water bath. THP-1 cells were mock- infected or T. asahii-infected at a multiplicity of infection (MOI) of $5: 1$.

\section{RNA extraction, library construction and sequencing}

Total RNA was extracted from both mock-infected and T. asahii-infected cells at $12 \mathrm{~h}$ time point by TRIzol reagent (Invitrogen, USA) according to the manufacturer's protocol. The concentration and purity of total RNA was analyzed by NanoDrop ND-2000 (Thermo Fisher Scientific, USA) and the integrity was evaluated by Agilent 2100 Bioanalyzer (Agilent Technologies, CA, USA). We constructed two types of libraries from six samples for sequencing as follows: (1) rRNA-depleted RNA library: The rRNA was removed in total of Loading [MathJax]/jax/output/CommonHTML/jax.js 
RNA (5 $\mu \mathrm{g})$ using a Ribo-off rRNA Depletion kit (Vazyme, China). After rRNA-depleted RNA purification, cDNA libraries were constructed using VAHTSTM Stranded mRNA-seq Library Prep Kit for Illumina® kits (Vazyme, China) according to the manufacturer's instructions. The Illumina Hiseq XTen platform (Sangon Biotech, China) was used to perform sequencing analysis. (2) miRNA library: Total RNA was collected and quantified by Qubit2.0 RNA detection kit, The 3' adapter and 5' adapter were ligated by using T4 RNA Ligase 2 (New England Biolabs, USA) and T4 RNA Ligase 1 (New England Biolabs, USA), respectively. Reverse transcription and PCR amplification of ligation products. The $140 \sim 150$ bp PCR products were enriched and quantified with Qubit 2.0 DNA kit. Finally the cDNA library was sequenced on the lllumina Hiseq XTen platform (Sangon Biotech, China).

\section{circRNA identification and characterization analysis}

The raw sequencing data were filtered by Trimmomatic software to obtain high quality clean data [16]. the quality control sequences were mapped to the human reference genome hg38 using BWA-MEM [17], Then subjected to CIRI2 to identify circRNAs [18]. The BEDtools was used to determine the source of circRNAs based on circRNAs position and gene annotation information [19].

\section{Abundance quantify and DE analysis}

mRNAs abundance were detected by StringTie software and normalized to transcripts per million (TPM) [20]. The formula for calculating TPM is as follows:

$T P M_{i}=\frac{X_{i}}{L_{i}} * \frac{1}{\sum_{j} \frac{X_{j}}{L_{j}}} * 10^{6}, \mathrm{X}_{\mathrm{i}}=\frac{\text { totalexonfragment }}{\text { reads }}, L_{i}=\frac{\text { exonlength }}{K B}$. The DESeq2 was used for DE analysis of mRNAs [21]. A q-value $<0.01, \| \log _{2}$ FoldChange (FC) $\mid>1$ and Mean TPM $>5$ in at least one group were set as thresholds for significant DE mRNAs. circRNAs expression were quantified based on the number of back-spliced junction read pairs and circRNAs length were calculated using Reads Per Kilobase of transcript per Million mapped reads (RPKM). The formula is shown as follows:

$\mathrm{RPKM}=\frac{\text { TotalExonReads }}{\text { MappedReads }(\text { millions }) * \text { ExonLength }(\mathrm{kb})} .|\mathrm{FC}|>1.5$ and $\mathrm{P}<0.05$ were considered

significantly DE. The miRNA level was analyzed and normalized counts to reads per million (RPM). The RPM was calculated as below: RPM $=\frac{\text { ExonMappedReads } * 10^{6}}{\text { TotalMappedReads }}$. The miRNAs were considered DE only when the $\left|\log _{2} F C\right|>1$ and $P<0.05$.

\section{RT-qPCR validation}

To verify the results of RNA-seq, we performed RT-qPCR analysis on some of the DE circRNAs, miRNAs and mRNAs. The total RNA was extracted from T. asahii infected and uninfected cells, and reverse transcribed to cDNA using a PrimeScript ${ }^{\text {TM }}$ RT reagent Kit with gDNA Eraser (Perfect Real Time) (Takara, Loading [MathJax]/jax/output/CommonHTML/jax.js 
Japan) according to the manufacturer's instructions. qPCR reactions were performed on $A B I$

StepOnePlusTM Real-Time PCR System (Applied Biosystems, USA). The divergent primers encompassing the back-splicing junctions for circRNAs and the convergent primers for mRNAs and miRNAs were synthesized by Sangon Biotech (China), and the sequences were shown in (Additional file 1: Table S1). $\beta$ actin was used as an internal control for circRNAs and mRNAs, and U6 served as the endogenous control for miRNAs. We used the $2^{-\triangle \triangle C T}$ method to analyze the data. Each experiment was repeated in triplicate.

\section{GO and KEGG pathway analysis}

GO enrichment and KEGG pathway analysis were performed to determine the role of the DE mRNAs and ceRNA network-associated target mRNAs. P-value $<0.05$ is a threshold indicate significant difference.

\section{ceRNA network analysis}

The circRNA-miRNA-mRNA network was constructed as the following standards: (1) DE circRNAs, miRNAs and mRNAs; (2) The expression of circRNA/mRNA and miRNA must have the opposite trend; (3) Pearson correlation coefficient $(r)>0.8$ was set as a threshold between circRNA and mRNA; (4) the coexpressed circRNA-mRNA pairs should have the same putative miRNA binding sites based on miRanda [22]. Cytoscape software (v3.8.0) was used to construct and visualize the ceRNA network [23].

\section{Cell transfection}

The sequence of hsa_circ_0065336 was cloned into overexpression vector pCD5-ciR (Geneseed, Guangzhou, China), miR-505-3p mimics (5'-CGUCAACACUUGCUGGUUUCCU-3') and miR-505-3p mimics negative controls (NC) (5'-ACUACUGAGUGACAGUAGA-3') were purchased from Ribobio (Guangzhou, China). THP-1 were transfected using Lipofectamine 2000 (Invitrogen, CA, USA), then co-cultured with heat-inactivated T. asahii for $12 \mathrm{~h}$ with a multiplicity of infection (MOI) of 5:1.

\section{Dual-luciferase assay}

The sequences of hsa_circ_0065336 and 3' UTR of PTPN11 with miR-505-3p binding site wild-type (WT) and mutant-type (MUT) were amplified and cloned into pmir-GLO (Promega, Madison, WI, USA). miR-505$3 p$ mimics or NC were co-transfected into 293T cells using Lipofectamine 2000 (Invitrogen, CA, USA). After $48 \mathrm{~h}$ post transfection. Luciferase activity was detected by Dual-Glo Luciferase Assay System (Promega, Madison, WI, USA) following the manufacturer's instructions.

\section{Statistical analysis}


The measured data were presented as the means \pm standard deviation (SD). Statistical significance of different groups were examined using Student's t-test. P-value $<0.05$ was defined as statistically significant.

\section{Results}

\section{Identification and characterization of circRNAs}

Six rRNA-depleted RNA libraries from $T$. asahii-infected and uninfected samples at $12 \mathrm{~h}$ were sequenced to analyse the profiles of circRNAs using RNA-sEq. The results showed that a total of 10539 unique circRNAs were identified, among which 4595, 3315 and 4456 circRNAs in three control samples, while 3422, 4423 and 4091 circRNAs in three T. asahii-infected samples (Fig. 1a), respectively. According to the position of circRNA on the genome, we found exonic circRNAs were the most abundant circRNAs with a number of 10388 (98.57\%), whereas intronic and intergenic circRNAs accounted only for 151 (1.43\%) (Fig. 1b). $82.69 \%$ of circRNAs were less than $2 \mathrm{k}$ bp and the average length was about $1.54 \mathrm{~kb}$ (Fig. 1c). The exons count distribution of circRNAs showed that most exonic circRNAs $(7739,73.43 \%)$ contained more than 2 exons (Fig. 1d). Further, analysis of circRNAs-host genes revealed that a great majority of hosting genes could produce multiple circRNAs isoforms (10539 circRNA candidates from 5551 parental genes) (Fig. 1e).

\section{Identification of DE circRNAs}

To investigate the DE profile of circRNAs in response to $T$. asahii infection, we employed hierarchical clustering analysis, the results indicated that the circRNA expression profiles were significantly changed after $T$. asahii infection. A total of 46 circRNAs were significantly dysregulated in THP-1 at $12 \mathrm{~h}$ post infection, with 19 circRNAs up-regulated and 27 circRNAs down-regulated (Fig. 2a, 2b and Additional file 1: Table S2). To verify the credibility of RNA-seq results, we randomly selected four significantly changed circRNAs (hsa_circ_0077736, hsa_circ_0065336, hsa_circ_0108123 and hsa_circ_0029624) for RT-qPCR verification. The RT-qPCR results exhibited an expression trend consisted with the RNA-seq results (Fig. 2c).

\section{Identification of DE mRNAs}

We also examined the change of mRNAs expression in the same condition. Cluster heatmap revealed that the differential expression patterns of mRNA in THP-1 after T. asahii infection compared with controls. A total of 95139 mRNA were identified and 412 mRNAs were DE between the two groups, of which, 276 mRNAs up-regulated and 136 mRNAs down-regulated (Fig. 3a, 3b and Additional file 1: Table S3). To further confirm the reliability of RNA-seq, four differentially expressed mRNAs (NECTIN2, MAPK14, CCL1 and CCL2) were randomly selected and validated with RT-qPCR. The results were highly consistent with 


\section{Identification of DE miRNAs}

We further analyzed the differentially expressed miRNAs from the same six samples. A total of 3218 miRNAs were detected, among which 47 miRNAs were significantly dysregulated between the two groups, including 32 miRNAs up-regulated and 15 miRNAs down-regulated (Fig. 4a, 4b and Additional file 1: Table S4). Four differentially expressed miRNAs (miR-4792, miR-193b-3p, miR-23b-3p and miR-133a-3p) were randomly selected and verified by RT-qPCR. As expected, the RT-qPCR results were consistent with that of RNA-seq (Fig. 4c).

\section{GO enrichment and KEGG analysis of DE mRNAs}

We performed GO enrichment and KEGG pathway analysis of the DE mRNAs. The GO enrichment analysis results showed that the DE mRNAs were highly enriched in some biological processes, such as leukocyte migration involved in inflammatory response, innate immune response in mucosa, leukocyte migration and phagocytosis (Fig. 5a). In addition, KEGG pathway analysis indicated that the plenty of mRNAs were primarily involved in Toll-like receptor signaling pathway, phagosome, endocytosis and TNF signaling pathway (Fig. 5b). These data implied that the cellular antifungal response was activated by $T$. ashiii infection.

\section{Analysis of circRNA-miRNA-mRNA regulatory network}

Recent studies showed that circRNAs can indirectly regulate gene expression by act as a miRNA sponge through a ceRNA mechanism. So we constructed a circRNA-miRNA-mRNA network according to the ceRNA hypothesis. The screening criterias are listed in the Methods. 44 cirRNA-miRNA-mRNA pathways were established, including five circRNAs, five miRNAs, and 42 mRNAs (Fig. 5c and Additional file 1: Table S5). Owing to there are so many mRNAs involved in the ceRNA network, we performed GO enrichment and KEGG pathway analysis of these above mRNAs. The GO enrichment analysis results showed that the DE mRNAs in the ceRNA network were mainly involved in leukocyte migration involved in inflammatory response, transmembrane receptor protein tyrosine kinase signaling pathway and phagocytosis (Fig. 5d). Furthermore, KEGG pathway analysis indicated that neurotrophin signaling pathway, lysosome, and natural killer cell mediated cytotoxicity were mostly involved (Fig. 5e).

\section{Verification of ceRNA network}

In order to demonstrate the authenticity of ceRNA network, we selected the predicted hsa_circ_0065336/miR-505-3p/PTPN11 pathway based on the literature and GO enrichment and KEGG pathway analysis in the previous step. We first measured the expression level of hsa_circ_0065336, miR505-3p and PTPN11 in THP-1 post infected with T. asahii at 0 h, 3 h, 6 h, 9 h, and $12 \mathrm{~h}$ time points, 
manner, and reached a peak at $12 \mathrm{~h}$. Whereas miR-505-3p was down-regulated starting at $3 \mathrm{~h}$ after infection, then maintained declining level at $9 \mathrm{~h}$ post infection, which is highly similar to the results of RNA-seq.

Bioinformatics prediction analysis showed that miR-505-3p targeted both hsa_circ_0065336 and PTPN11 based on miRanda database. In order to confirm that, the full-length of hsa_circ_0065336-WT and MUT were constructed and subcloned into dual-luciferase report vector pmir-GLO, then co-transfected with miR505-3p mimics or NC into 293T cells. The results showed that hsa_circ_0065336-WT with miR-505-3p mimics could significantly decrease the luciferase activity, whereas hsa_circ_0065336-MUT was not affected. As well, the luciferase activity of PTPN11 3'UTR-WT was significantly decreased by miR-505-3p mimics compared with control groups.

To further verified the hsa_circ_0065336/miR-505-3p/PTPN11 pathway in THP-1 cells after T. asahii infection, we transfected the hsa_circ_0065336 over expression vector into THP-1 cells and subsequently infected with $T$. asahii for $12 \mathrm{~h}$. The results indicated that overexpression of hsa_circ_0065336 could significant elevated PTPN11 level compared with control. In addition, the increase of PTPN11 induced by hsa_circ_0065336 overexpression could be reversed by miR-744-3p mimics. Taken together, these data identified the hsa_circ_0065336/miR-505-3p/PTPN11 pathway in THP-1 upon T. asahii infection.

\section{Discussion}

Recently, several studies have reported the cricRNA expression profiles and ceRNA network in viral and bacteria infection diseases[24-27]. Nevertheless, the expression profiles and potential roles of circRNAs in fungi infections have not been explored.

In this study, we systematically investigated circRNA, miRNA, and mRNA expression profiles in $T$. asahiiinfected THP-1 cells compared with uninfected samples. The characteristic of circRNA expression, type, length and host gene number were similar with previous reports[28, 29]. We found a total of $46 \mathrm{DE}$ circRNAs, 412 DE mRNAs and 47 DE miRNAs upon T. asahii infection after $12 \mathrm{~h}$, of these mRNAs, GO enrichment and KEGG pathway analysis showed that they were primarily enriched in immune-related pathways, suggesting that $T$. ashii infection could activate a strong antifungal response, contributing to fungi pathogenic progression.

The ceRNA hypothesis was first proposed by Salmena et al [30] in 2011 described how mRNAs, transcribed pseudogenes, and IncRNAs "talk" to each other using MREs as letters of a new language. Many studies have identified that circRNAs could act as ceRNAs to sponge miRNAs, indirect regulating the expression of genes and participate in the occurrence and development of various diseases, especially cancer. Since no data about circRNA-associated ceRNA have been reported in fungal infection, we discovered a systematic circRNA-miRNA-mRNA regulatory network after $T$. asahii infection based on the RNA-seq and ceRNA rules. Five circRNAs, five miRNAs, and 42 mRNAs were screened and constructed the ceRNA regulatory network. Further more, we performed GO and KEGG analysis on the 42 predicted 
showed that many host immune response pathways were involved, including inflammatory response, phagocytosis and neurotrophin signaling pathway, indicating that circRNAs may be the principal regulators of host response during $T$. asahii infection.

Moreover, We selected hsa_circ_0065336/miR-505-3p/PTPN11 pathway to verified the reliability of ceRNA network using RT-qPCR, dual luciferase reporter assay and overexpression experiment. PTPN11 is a cytoplasmic tyrosine phosphatase that participate the signaling of growth factors, cytokines and hormones. Recent study found that PTPN11 is crucial for the induction of pro-inflammatory cytokines and control candida albicans infection through Dectin-1/PTPN11/Syk/NF-kB pathway [31]. miR-505-3p has been showed to be down-regulated in several autoimmune disease, including primary biliary cirrhosis and inflammatory bowel disease [32, 33]. Rafael et al. [34] reported that miR-505-3p was decreased in familial hypercholesterolemia, which could up-regulated several chemokine receptor through target RUNX1 transcription factor in macrophages. hsa_circ_0065336 has been reported expression in brain and several cell types, but no study has assessed its function in any disease or physiological process. Here, we identified the hsa_circ_0065336 and PTPN11 expression were simultaneously up-regulated, whereas miR-505-3p expression was down-regulated in T. asahii infected THP-1 cells. Dual-luciferase reporter assay demonstrated that miR-505-3p can direct target hsa_circ_0065336 and PTPN11. Moreover, function assay revealed that PTPN11 expression was up-regulated by hsa_circ_0065336 while inhibited by miR-505-3p. Therefore, the hsa_circ_0065336/miR-505-3p/PTPN11 regulatory axis was found and verified for the first upon $T$. asahii infection.

\section{Conclusions}

As far as we know, this is the first report to conjoint analyze the expression profiles of circRNA, miRNA and mRNA upon fungal infection in mammal cells. We construct a circRNA-associated ceRNA regulatory network, which mainly involved in fungal-host immune response. In conclusion, this study could provide novel insight for clarifying the pathogenesis of T. asahii infection.

\section{Abbreviations}

ceRNA: competing endogenous RNA; circRNA: circular RNA; DE: differentially expressed; GO: Gene Ontology; KEGG: Kyoto Encyclopedia of Genes and Genomes; miRNA: microRNA; MOI: multiplicity of infection; MREs: miRNA response elements; MT: wild type; MUT: mutant type; NC: negative controls; PMA: Phorbol 12-myristate 13-acetate; RNA-seq: RNA sequencing; RPKM: Reads Per Kilobase of transcript per Million mapped reads; RPM: reads per million; RT-qPCR: real-time quantitative PCR; T. asahii: Trichosporon asahii; TPM: transcripts per million.

\section{Declarations}

\section{Acknowledgement}


The authors express their gratitude to all of the laboratory members for their assistance.

\section{Authors' contributions}

MWZ and ZHZ conducted the experiments and wrote the manuscript. ZKX, YX, WTL and JHA collected and analyzed the data. RYY supervised the project. All authors read and approved the final manuscript.

\section{Funding}

This work was supported by the National Natural Science Foundation of China (NO. 81571972, 81471928) and the National Science Foundation of Beijing, China (NO. 7202201).

\section{Availability of data and materials}

The datasets used and/or analysed during the current study are available from the corresponding author on reasonable request.

\section{Ethics approval and consent to participate}

Not applicable

\section{Consent for publication}

Not applicable

\section{Competing interests}

The authors declare that they have no competing interests.

\section{References}

1. Marine M, Brown NA, Riano-Pachon DM, Goldman GH. On and Under the Skin: Emerging Basidiomycetous Yeast Infections Caused by Trichosporon Species. PLoS Pathog. 2015;11(7):e1004982.

2. Liao Y, Lu X, Yang S, Luo Y, Chen Q, Yang R. Epidemiology and Outcome of Trichosporon Fungemia: A Review of 185 Reported Cases From 1975 to 2014. Open Forum Infect Dis. 2015;2(4):ofv141.

3. Colombo AL, Padovan AC, Chaves GM. Current knowledge of Trichosporon spp. and 
4. Kristensen LS, Andersen MS, Stagsted LVW, Ebbesen KK, Hansen TB, Kjems J. The biogenesis, biology and characterization of circular RNAs. Nature reviews Genetics. 2019;20(11):675-91.

5. Sanger HL, Klotz G, Riesner D, Gross HJ, Kleinschmidt AK. Viroids are single-stranded covalently closed circular RNA molecules existing as highly base-paired rod-like structures. Proceedings of the National Academy of Sciences of the United States of America. 1976;73(11):3852-56.

6. Cocquerelle C, Mascrez B, Hétuin D, Bailleul B. Mis-splicing yields circular RNA molecules. FASEB journal : official publication of the Federation of American Societies for Experimental Biology. 1993;7(1):155-60.

7. Vo JN, Cieslik M, Zhang Y, Shukla S, Xiao L, Zhang Y, et al. The Landscape of Circular RNA in Cancer. Cell. 2019;176(4):869-81.

8. Lu S, Yang X, Wang C, Chen S, Lu S, Yan W, et al. Current status and potential role of circular RNAs in neurological disorders. Journal of neurochemistry. 2019;150(3):237-48.

9. Aufiero S, Reckman YJ, Pinto YM, Creemers EE. Circular RNAs open a new chapter in cardiovascular biology. Nature reviews Cardiology. 2019;16(8):503-14.

10. Shan K, Liu C, Liu B-H, Chen X, Dong R, Liu X, et al. Circular Noncoding RNA HIPK3 Mediates Retinal Vascular Dysfunction in Diabetes Mellitus. Circulation. 2017;136(17):1629-42.

11. Zhou Z, Sun B, Huang S, Zhao L. Roles of circular RNAs in immune regulation and autoimmune diseases. Cell death \& disease. 2019;10(7):503.

12. Ha M, Kim VN. Regulation of microRNA biogenesis. Nature reviews Molecular cell biology. 2014;15(8):509-24.

13. Ma S-Q, Xu X-X, He Z-Z, Li X-H, Luo J-M. Dynamic changes in peripheral blood-targeted miRNA expression profiles in patients with severe traumatic brain injury at high altitude. Military Medical Research. 2019;6(1):12.

14. Croston TL, Lemons AR, Beezhold DH, Green BJ. MicroRNA Regulation of Host Immune Responses following Fungal Exposure. Frontiers in immunology. 2018;9:170.

15. Qi X, Zhang D-H, Wu N, Xiao J-H, Wang X, Ma W. ceRNA in cancer: possible functions and clinical implications. Journal of medical genetics. 2015;52(10):710-18.

16. Bolger AM, Lohse M, Usadel B. Trimmomatic: a flexible trimmer for Illumina sequence data. Bioinformatics. 2014;30(15):2114-20.

17. <Aligning sequence reads, clone sequences and assembly contigs with BWA-MEM.pdf>.

18. Gao Y, Zhang J, Zhao F. Circular RNA identification based on multiple seed matching. Briefings in bioinformatics. 2018;19(5):803-10.

19. Quinlan AR, Hall IM. BEDTools: a flexible suite of utilities for comparing genomic features. Bioinformatics (Oxford, England). 2010;26(6):841-42.

20. Pertea M, Pertea GM, Antonescu CM, Chang TC, Mendell JT, Salzberg SL. StringTie enables improved reconstruction of a transcriptome from RNA-seq reads. Nat Biotechnol. 2015;33(3):290-5. 
21. Love MI, Huber W, Anders S. Moderated estimation of fold change and dispersion for RNA-seq data with DESeq2. Genome Biol. 2014;15(12):550.

22. John B, Enright AJ, Aravin A, Tuschl T, Sander C, Marks DS. Human MicroRNA targets. PLoS biology. 2004;2(11):e363.

23. Shannon P, Markiel A, Ozier O, Baliga NS, Wang JT, Ramage D, et al. Cytoscape: a software environment for integrated models of biomolecular interaction networks. Genome research. 2003;13(11):2498-504.

24. Lu S, Zhu N, Guo W, Wang X, Li K, Yan J, et al. RNA-Seq Revealed a Circular RNA-microRNA-mRNA Regulatory Network in Hantaan Virus Infection. Frontiers in cellular and infection microbiology. 2020;10:97.

25. Ma X, Zhao X, Zhang Z, Guo J, Guan L, Li J, et al. Differentially expressed non-coding RNAs induced by transmissible gastroenteritis virus potentially regulate inflammation and NF-KB pathway in porcine intestinal epithelial cell line. BMC genomics. 2018;19(1):747.

26. Shi J, Hu N, Mo L, Zeng Z, Sun J, Hu Y. Deep RNA Sequencing Reveals a Repertoire of Human Fibroblast Circular RNAs Associated with Cellular Responses to Herpes Simplex Virus 1 Infection. Cellular physiology and biochemistry : international journal of experimental cellular physiology, biochemistry, and pharmacology. 2018;47(5):2031-45.

27. Zhang $Y$, Zhang H, An M, Zhao B, Ding H, Zhang Z, et al. Crosstalk in competing endogenous RNA networks reveals new circular RNAs involved in the pathogenesis of early HIV infection. Journal of translational medicine. 2018;16(1):332.

28. Zheng Q, Bao C, Guo W, Li S, Chen J, Chen B, et al. Circular RNA profiling reveals an abundant circHIPK3 that regulates cell growth by sponging multiple miRNAs. Nature communications. 2016;7:11215.

29. Shi J, Hu N, Li J, Zeng Z, Mo L, Sun J, et al. Unique expression signatures of circular RNAs in response to DNA tumor virus SV40 infection. Oncotarget. 2017;8(58):98609-22.

30. Salmena L, Poliseno L, Tay Y, Kats L, Pandolfi PP. A ceRNA hypothesis: the Rosetta Stone of a hidden RNA language? Cell. 2011;146(3):353-58.

31. Deng Z, Ma S, Zhou H, Zang A, Fang Y, Li T, et al. Tyrosine phosphatase SHP-2 mediates C-type lectin receptor-induced activation of the kinase Syk and anti-fungal TH17 responses. Nature immunology. 2015;16(6):642-52.

32. Pekow JR, Kwon JH. MicroRNAs in inflammatory bowel disease. Inflammatory bowel diseases. 2012;18(1):187-93.

33. Ninomiya M, Kondo Y, Funayama R, Nagashima T, Kogure T, Kakazu E, et al. Distinct microRNAs expression profile in primary biliary cirrhosis and evaluation of miR 505-3p and miR197-3p as novel biomarkers. PloS one. 2013;8(6):e66086.

34. Escate R, Mata P, Cepeda JM, Padreó T, Badimon L. miR-505-3p controls chemokine receptor upregulation in macrophages: role in familial hypercholesterolemia. FASEB journal : official publication Loading [MathJax]/jax/output/CommonHTML/jax.js for Experimental Biology. 2018;32(2):601-12.

Page $12 / 18$ 
Figures
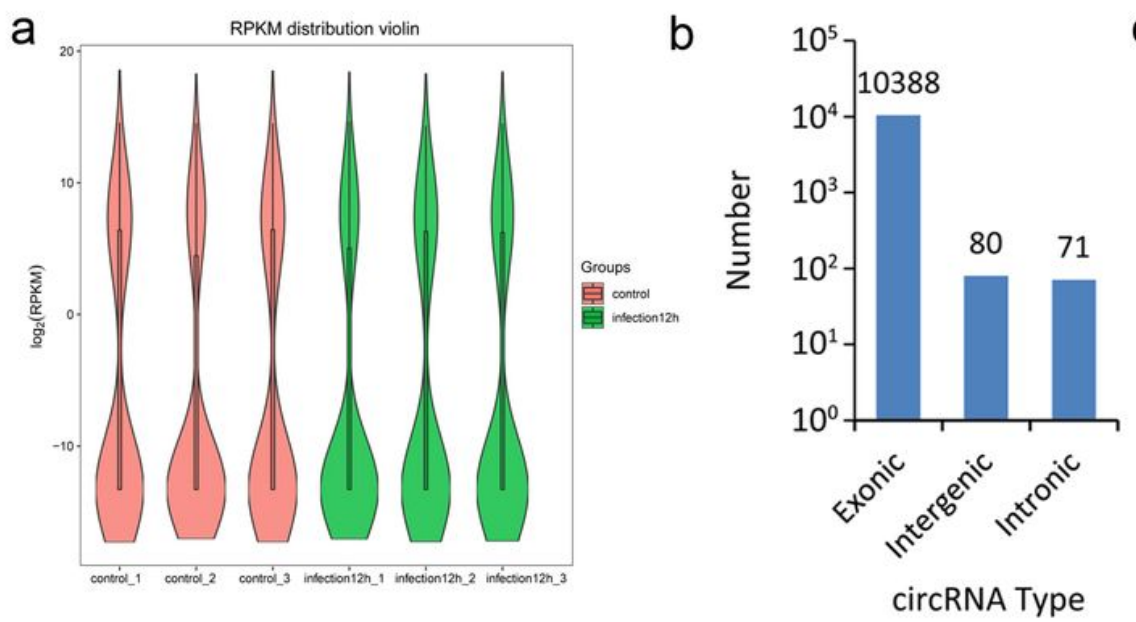
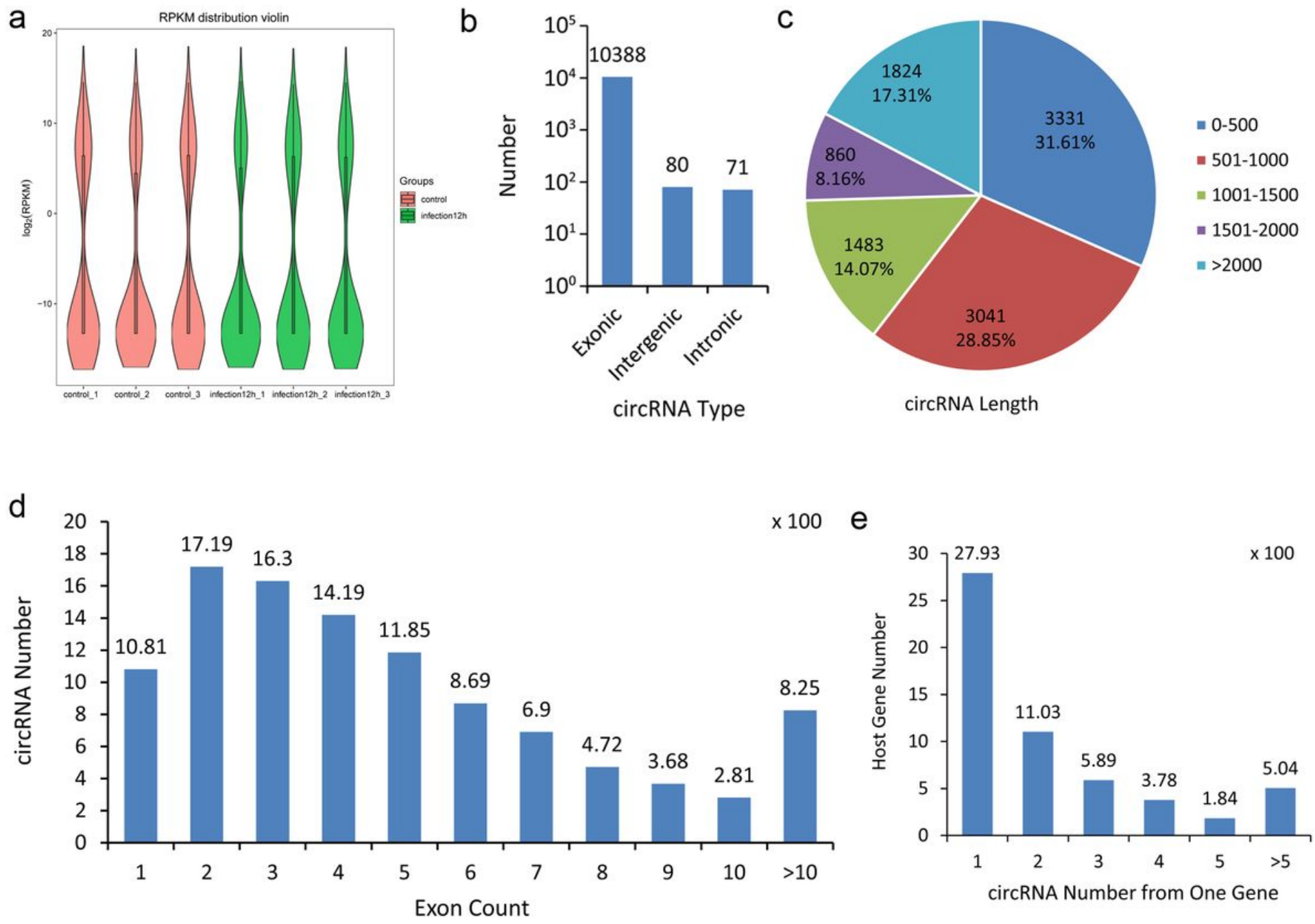

\section{Figure 1}

profiles and characteristics of circRNAs. a. Violin plot shows the expression level of circRNAs in each sample. b. The number of circRNAs based on their genomic position. c. The length distribution of circRNA candidates. $d$. The counts of exon from one circRNA. e. The number of circRNAs transcribed from one gene. 
a

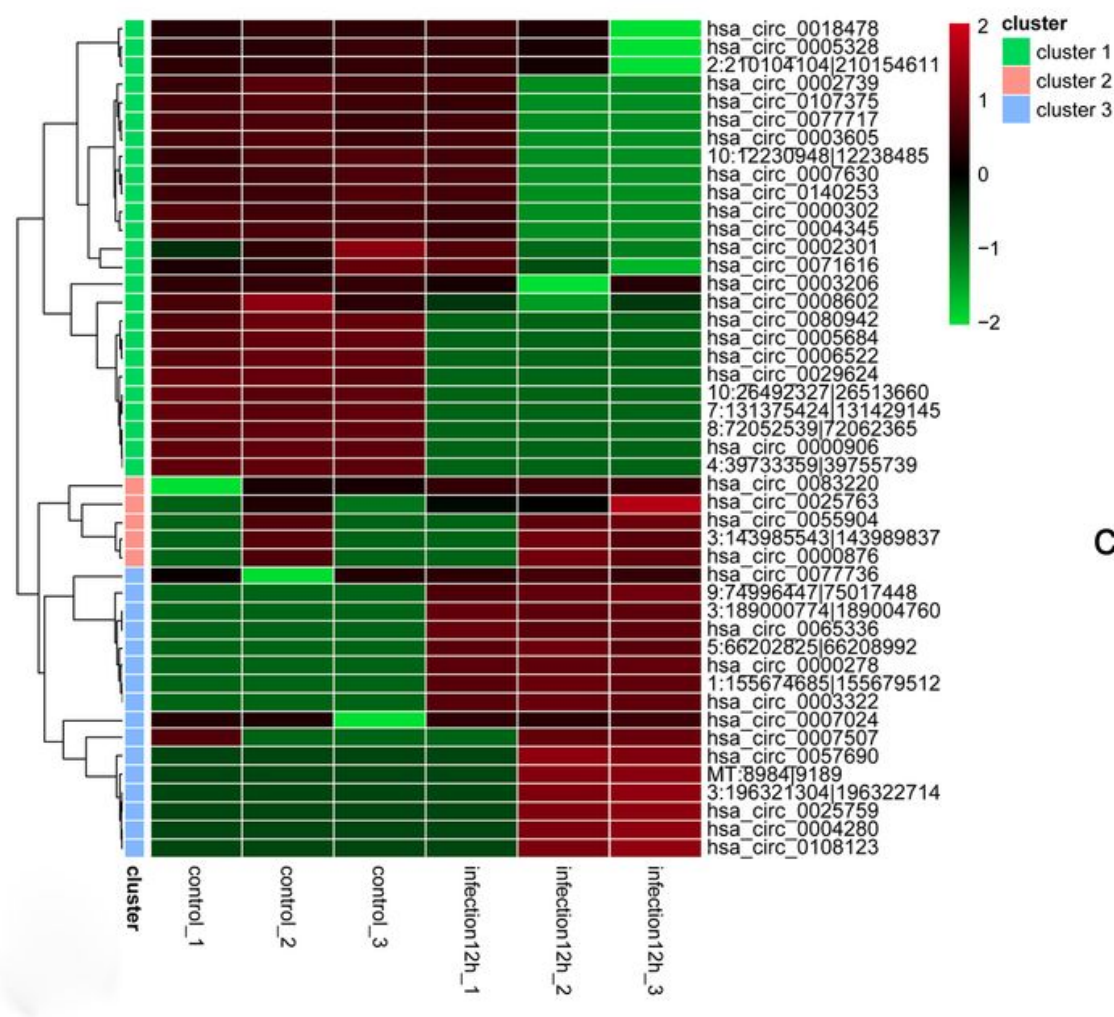

b

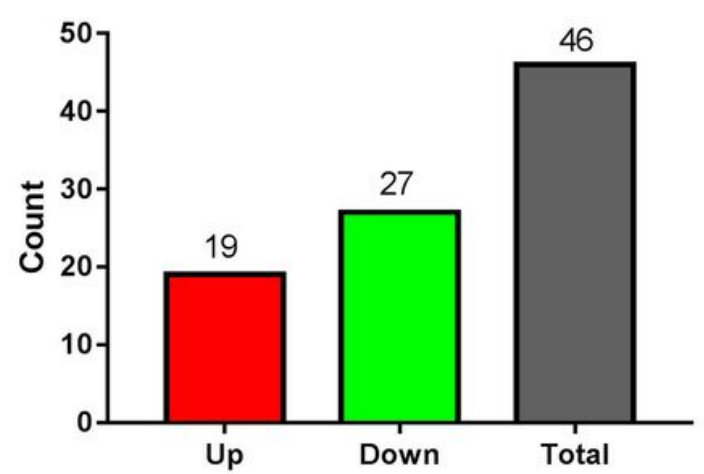

C

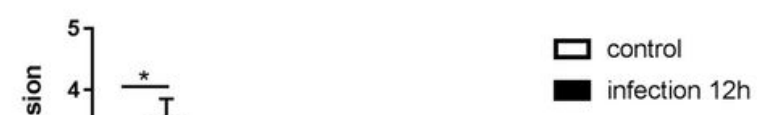

Figure 2

Identification of DE circRNAs after T.asahii infection. a. Heatmap and clustering analysis of DE circRNAs induced by T. asahii in THP-1 cells. b. DE circRNAs number. c. Verification of dysregulated circRNAs by RT-qPCR. *P $<0.05 ; * \star P<0.01 ; * \star \star P<0.001$ was considered as statistically significant. 
a

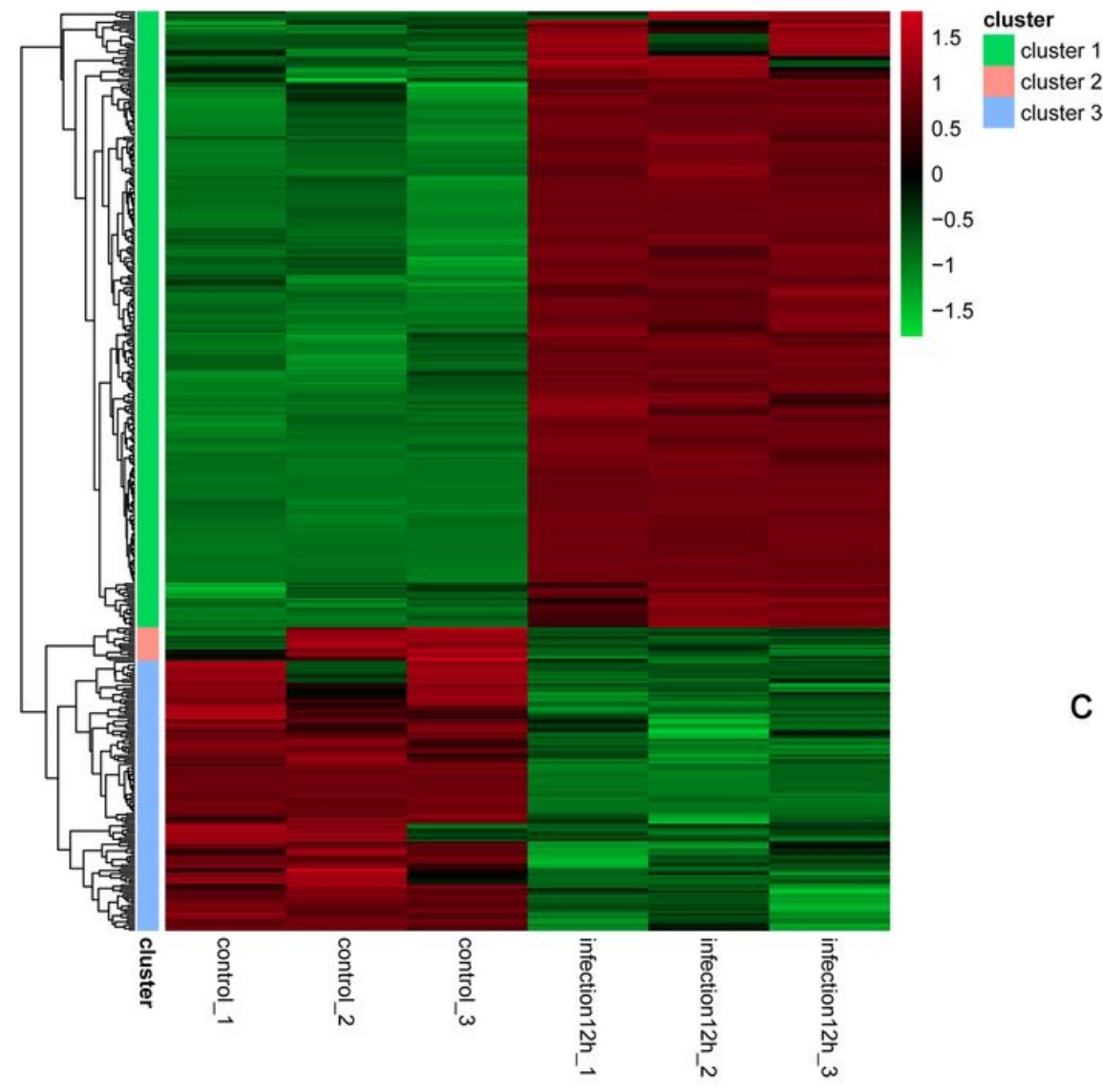

b

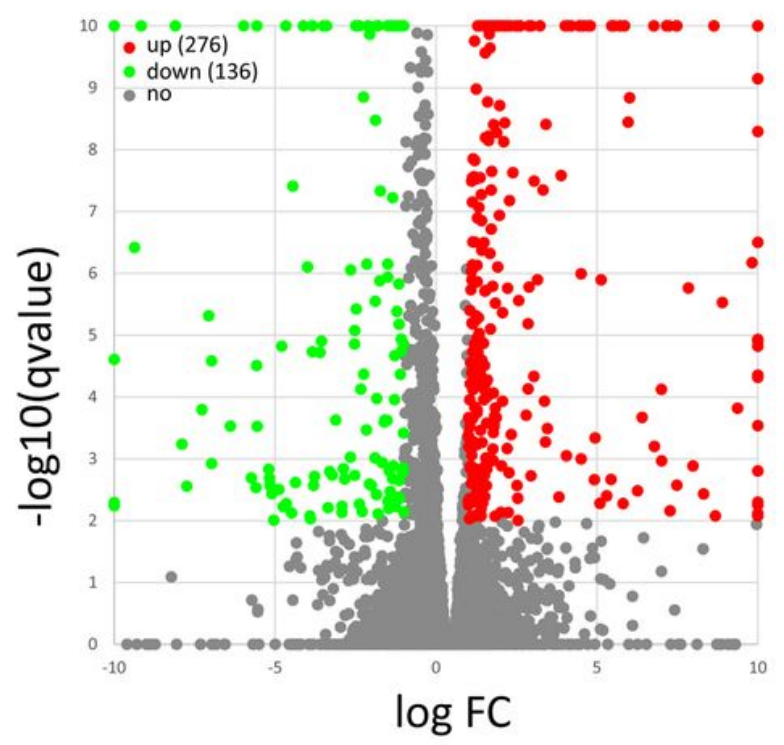

C

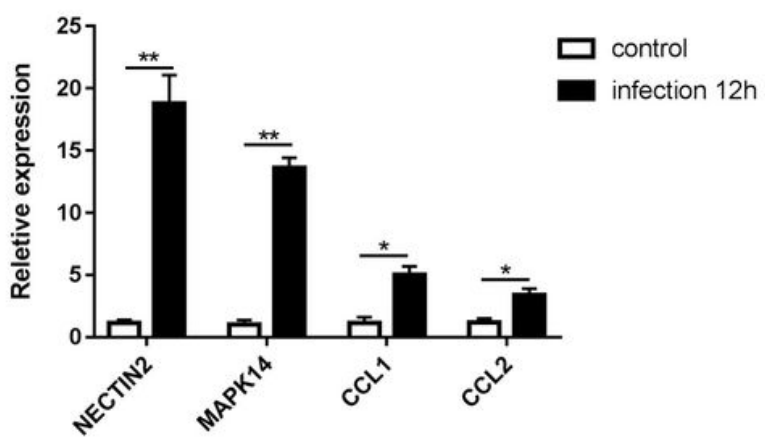

\section{Figure 3}

Identification of DE mRNAs in the T. asahii-infected group and the uninfected group. a. Heatmap and clustering analysis of DE mRNAs. b. Volcano plot of DE mRNAs in the $12 \mathrm{~h}$ post infection against control groups. c. Verification of DE mRNAs by RT-qPCR. ${ }^{*} P<0.05 ; * \star P<0.01$; ${ }^{* \star * P}<0.001$ was considered as statistically significant. 
a

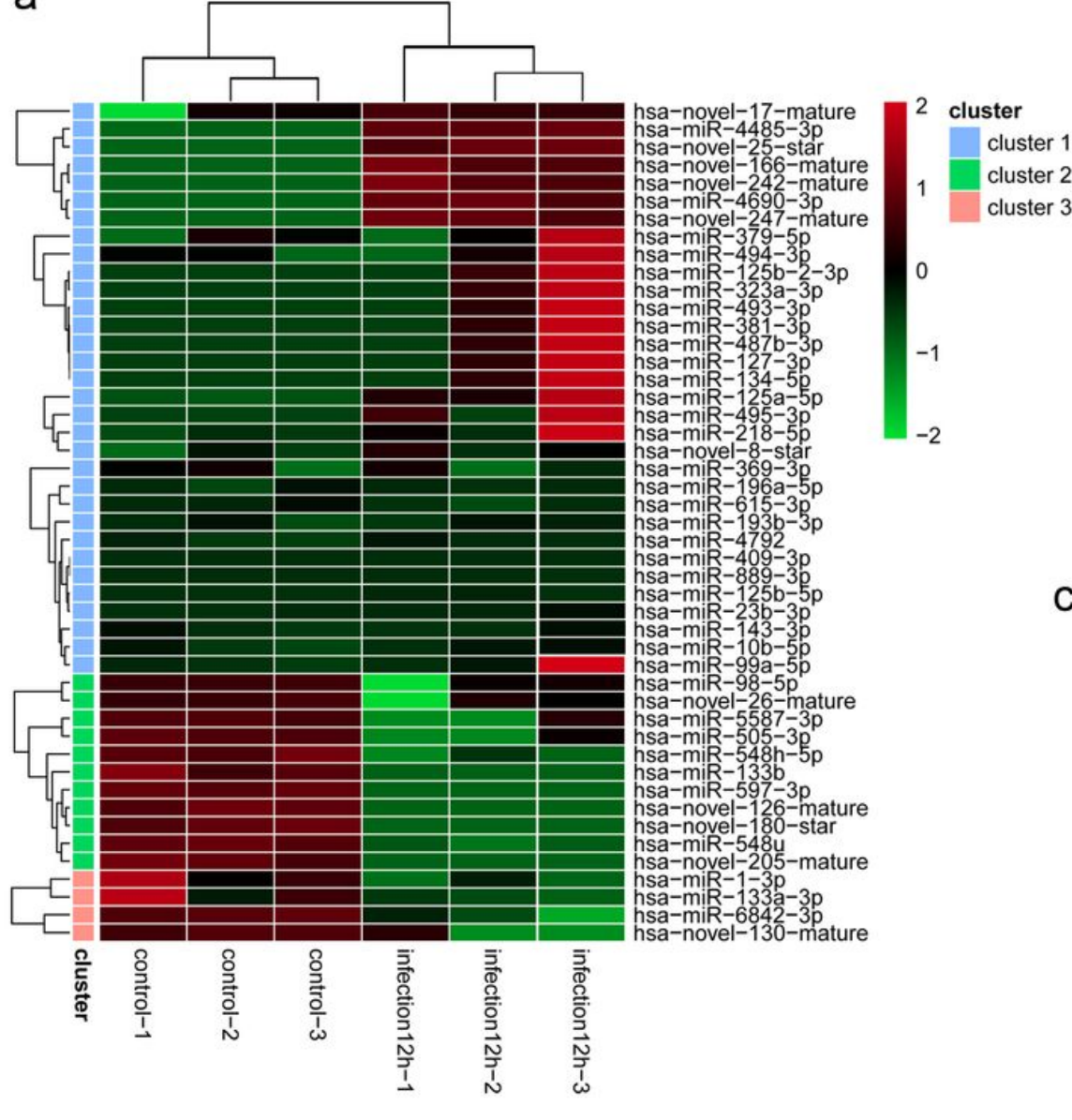

b

\section{miRNA}

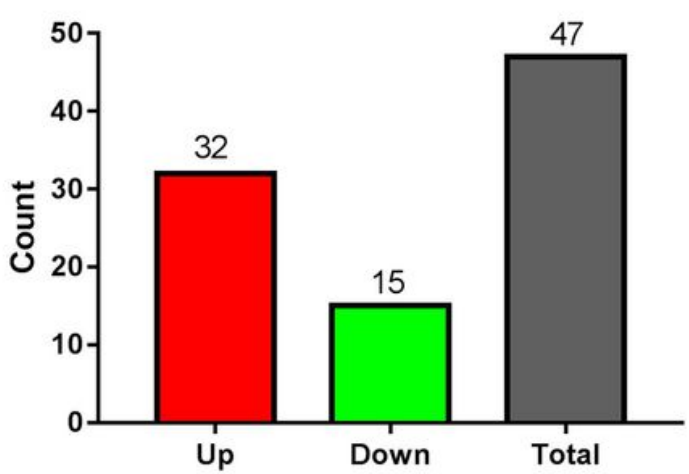

C

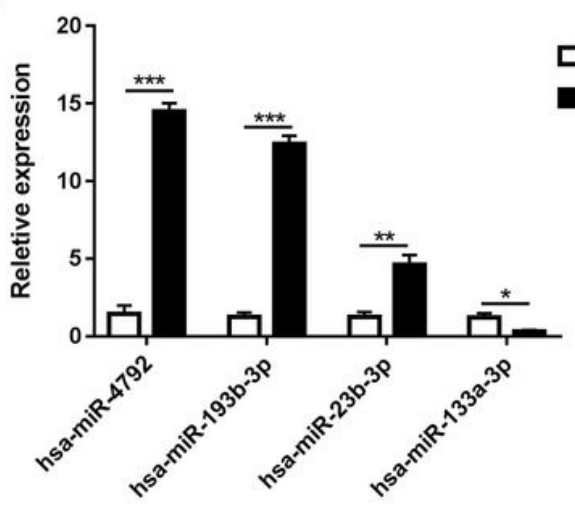

Figure 4 
a

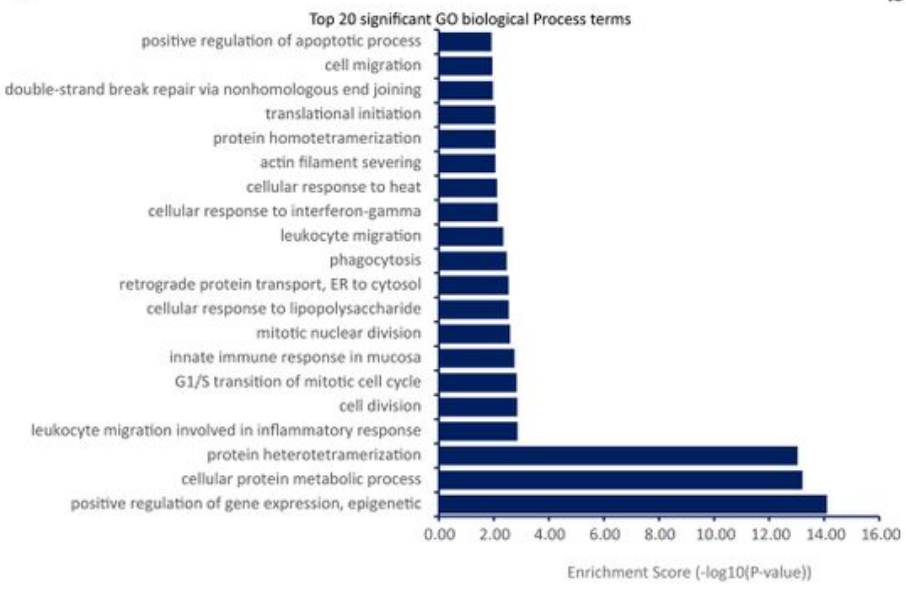

b

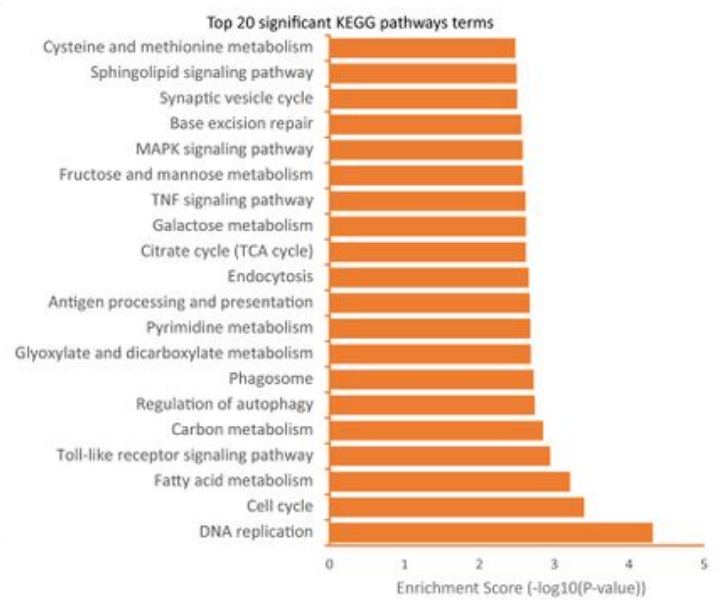

C

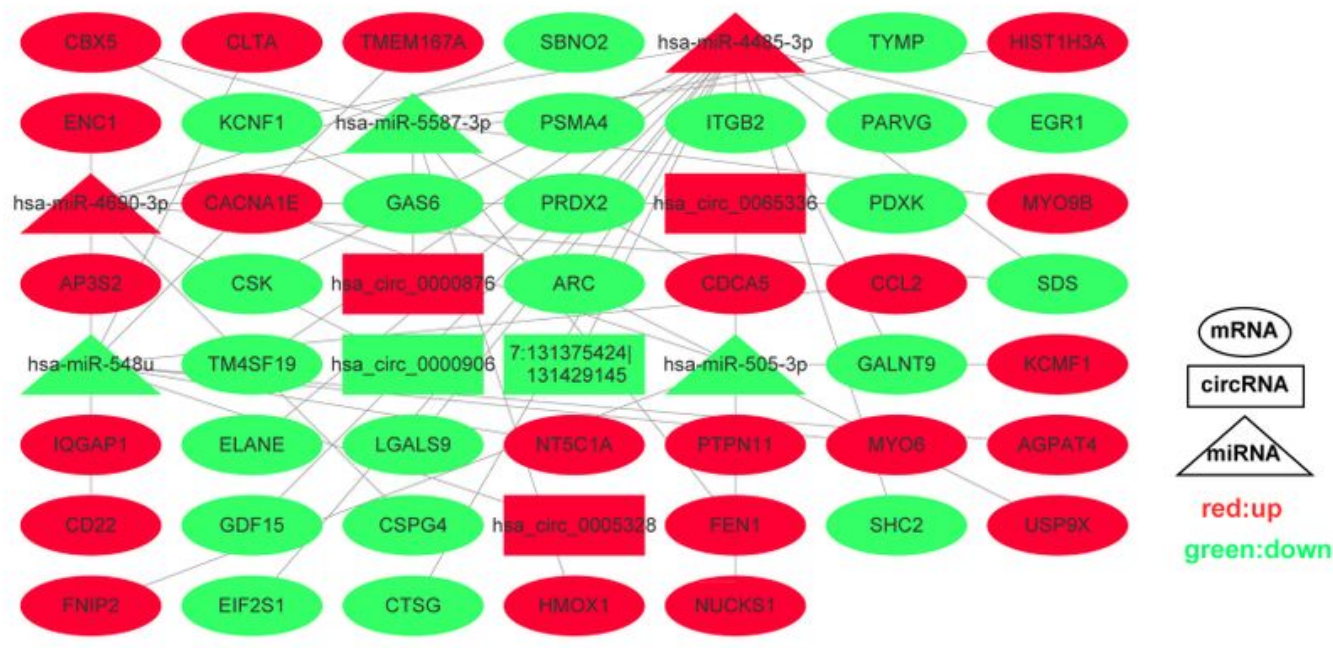

d

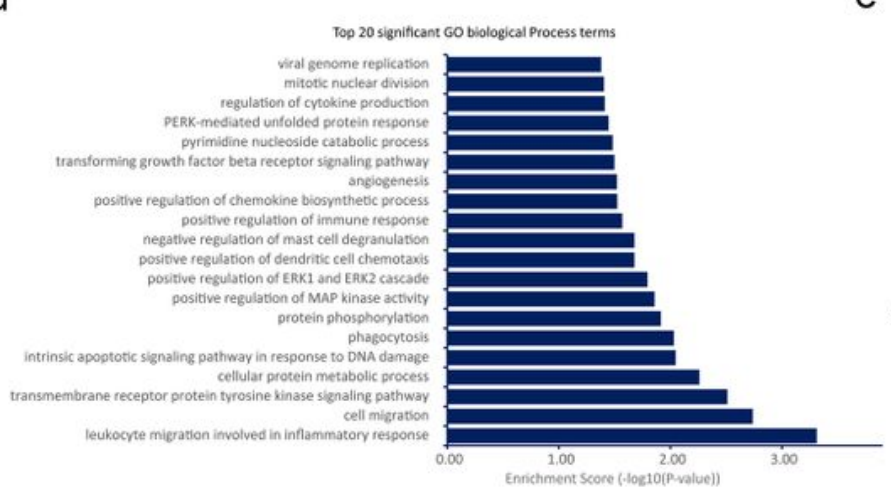

e

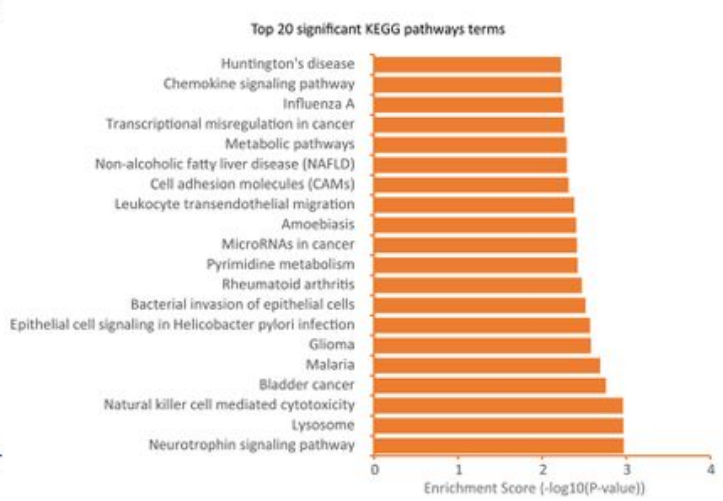

\section{Figure 5}

Function analysis and circRNA-miRNA-mRNA interaction network. (a) and (b) Top 20 GO biological process terms and KEGG pathways terms of DE mRNAs, respectively. c. ceRNA co-expression network. (d) and (e) Top 20 GO biological process terms and KEGG pathways terms of the targeting mRNAs of the ceRNA networks. 
a

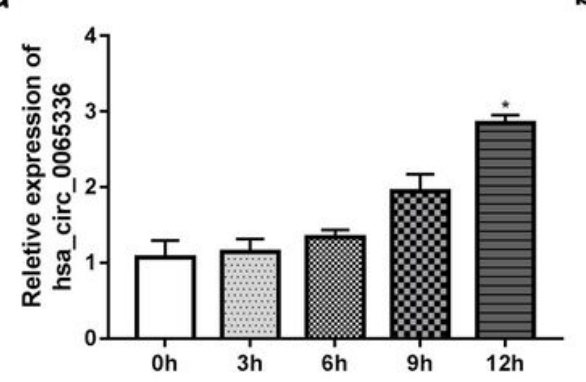

d hsa_circ_0065336 WT 5' AAGAGCACUCAGACUGUUGACA 3' miR-505-3p 3' UCCUUUGGUCGUUCACAACUGC 5' hsa_circ_0065336 MUT 5' AAGAGCACUCAGACACAACUGC 3'

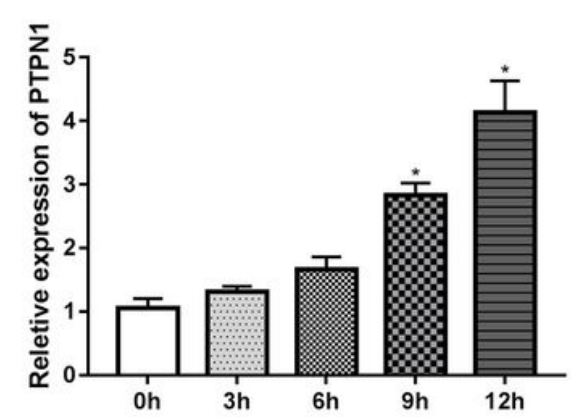

C

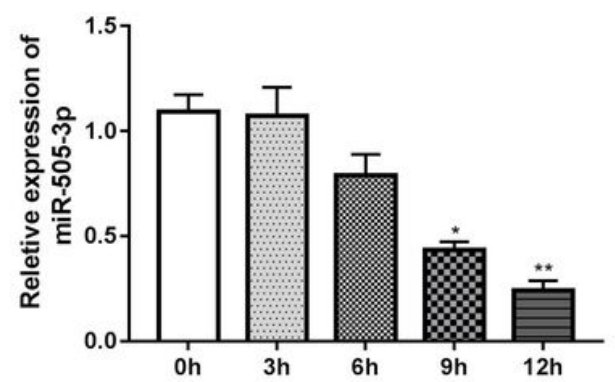

e

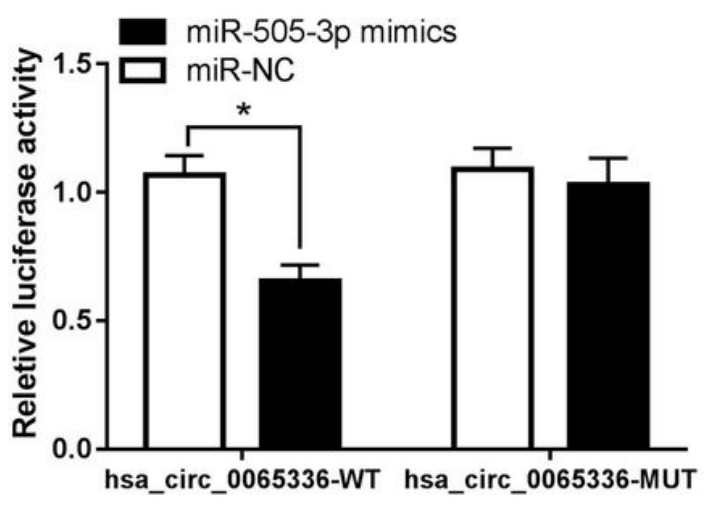

$\mathrm{h}$
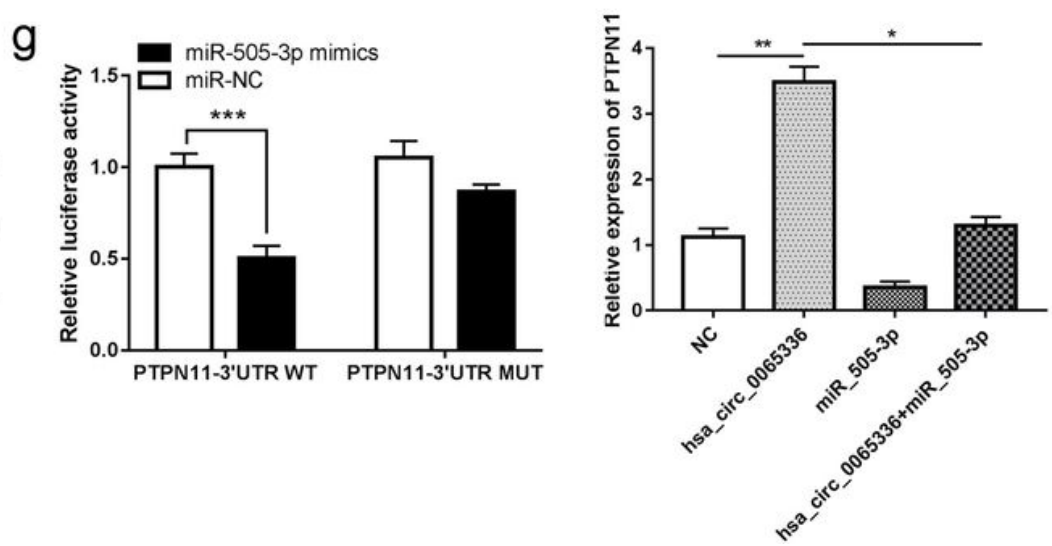

Figure 6

\section{Supplementary Files}

This is a list of supplementary files associated with this preprint. Click to download.

- Additionalfile1TableS1.xlsx

- Additionalfile1TableS4.xIsx

- Additionalfile1Tables2.xlsx

- Additionalfile1Tables5.xlsx

- Additionalfile1TableS3.xIsx 